\title{
Improved Color QR Codes for Real Time Applications with High Embedding Capacity
}

\author{
M. Ramya \\ PG scholar \\ Kalaignar Karunanidhi Institute of Technology \\ Coimbatore, India
}

\author{
M. Jayasheela \\ $\mathrm{HOD} / \mathrm{PG}$ \\ Kalaignar Karunanidhi Institute of Technology \\ Coimbatore, India
}

\begin{abstract}
A QR code is a special type of barcode that can encode information like numbers, letters, and any other characters. The capacity of a given QR code depends on the version and error correction level, as also the data type which are encoded. A QR code framework for mobile phone applications by exploiting the spectral diversity afforded by the cyan (C), magenta (M), and yellow (Y) print colorant channels commonly used for color printing and the complementary red $(\mathrm{R})$, green $(\mathrm{G})$, and blue (B) channels, which captures the color images had been proposed. Specifically, this spectral diversity to realize a threefold increase in the data rate by encoding independent data the $\mathrm{C}, \mathrm{Y}$, and $\mathrm{M}$ channels and decoding the data from the complementary R, G, and B channels. In most cases ReedSolomon error correction codes will be used for generating error correction codeword's and also to increase the interference cancellation rate. Experimental results will show that the proposed framework successfully overcomes both single and burst errors and also providing a low bit error rate and a high decoding rate for each of the colorant channels when used with a corresponding error correction scheme. Finally proposed system was successfully synthesized using QUARTUS II EDA tools.
\end{abstract}

\section{Keywords}

Reed Solomon (RS), Field programmable gate array (FPGA).

\section{INTRODUCTION}

Bar code is one of the existing system which is very fast in scanning and more accurate when compared to other cding systems.. Barcode enables tracking in an efficient manner. The speed of scanning the barcode system is very high when compared to manual data entry method. 2D barcode is developed from 1D barcode and the information that are encoded will be stored in vertical direction as well as in horizontal direction. The advantages of $2 \mathrm{D}$ barcodes includes: less area, high embedding capacity, higher density, higher error error detection level. The advanced level of barcode is the stacked barcode which are stacked one upon another. These barcodes are printed in a rectangular shape which can able to achieve area. A special type of stack two-dimensional barcode is PDF417. The advanced level of barcode is the QR code which is a advanced matrix two-dimensional barcode. These QR codes can able to detect the errors more effectively than other codes such 1D and 2D barcodes. The embedding capacity of the QR code is very less when compared to the other barcodes.

\section{Features of QR Code}

QR Code (Quick Response Code) There four levels of error correction, and the maximum symbol size can encoding 7089 numeric data or 4296 alphanumeric data [1]. The highest error correction level is upto $30 \%$ of code words of the symbol. The advanced features of $\mathrm{QR}$ code are:
1) High embedding Capacity.
2) High speed scanning
3) Represented by two bits of data.
4) It can be readable from any direction from 360 degree.

\section{PER-COLORANT DATA ENCODING}

Each QR Code symbol consists of an encoding region, alignment patterns and function patterns, as shown in Fig. 1. Function patterns includes finder, separator. These are not used for encoding the data. These are detected with several versions from version 1 to version 40 .

The encode steps of QR Code are shown below. Firstly input data is encoded formed bit stream in an efficient mode. The bit streams which are obtained by encoding the data are divided into codewords. These codewords are again divided intosets of blocks blocks and error correction level is added to all the set of blocks. These codewords are masked with mask pattern. Finally function patterns and separators are are added into the QR symbol. A QR Code symbol is formed as shown in the figure 1.

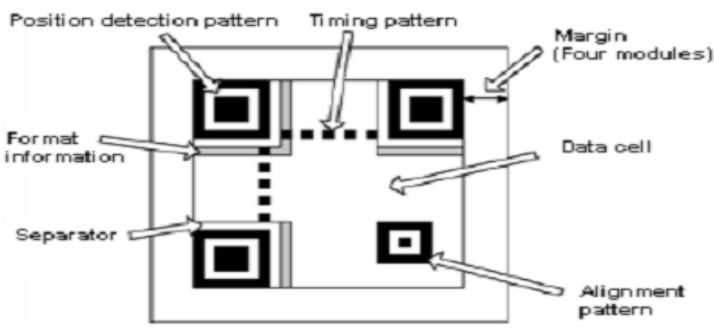

Fig 1: Structure of $Q R$ code

Compared with 1D barcode, the 2D barcodes has a much larger capacity which can hold more data than $1 \mathrm{D}$ barcode. A QR code capcity is upto 4296 letters, and 2953 binary code word data and 7089 digits.

\section{PROPOSED SYSTEM}

Color printing uses cyan, yellow. magenta for color reproduction in printing. Color capture devices uses Red, green, blue sensing channels. These are complementary to cyan , yellow , magenta colors. Recently the study of color barcodes provides the information for increase in embedding capacity than monochrome barcodes. All the data are initially extracted from red, green, blue channels. The CMY colorant channel are also extracted from RGB model parameters. The combination of all these colors provides the color code with high embedding capacity. The proposed system for Color QR code generation is shown in the Figure 2. 


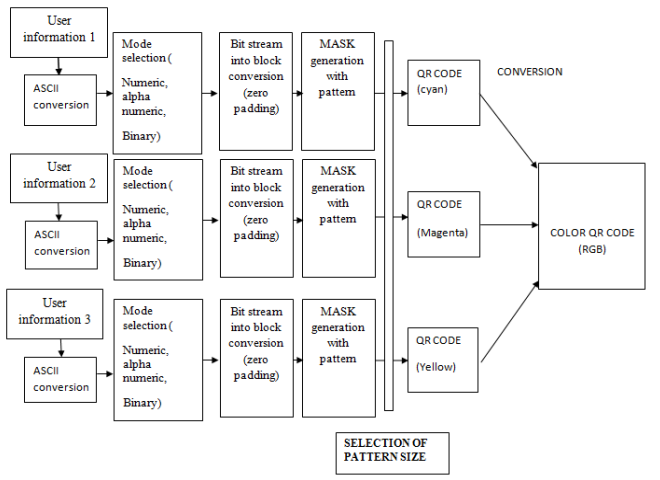

Fig 2: Proposed system for color QR code generation

Consider the input as an alpha numeric value. Initially the data is given as an input. The information encoded by a $\mathrm{QR}$ code may be made up of four standardized types "modes" of data such as numeric, alphanumeric, byte / binary or through some automatic extensions, virtually is can be any type of data.

All these datas are collected and converted into ASCII values. Then the data is divided into groups of two elements. Each ASCII value is added with next data's ASCII values. All the data's values are added accordingly and grouped into 11 bits. These bits of data are segmented into blocks.

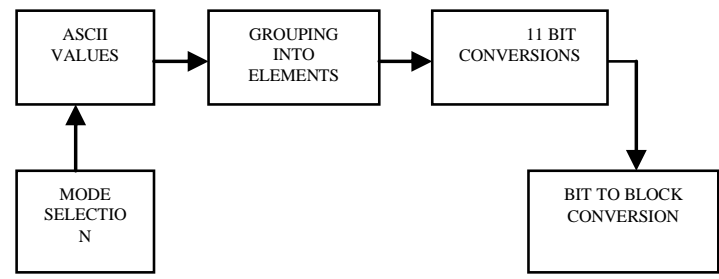

Fig 3: Bit To Block Conversion

\section{GENERATING THE COLOR QR CODE}

The proposed system is composed of two main coder for converting the obtained data into stream of datas. The two main coders are:

Reed Solomon coder is used in many burst error correction. The input data are arranged in block format and parity blocks are added with each set. In order to achieve variable code rate scaling is performed on the output of the convolution encoder. By concatenating the output of both reed Solomon coder and convolution coder, the input provided is converted into stream of data.

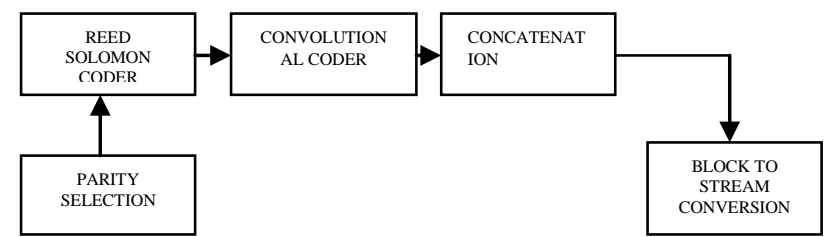

Fig 4: QR Code Generation

Convolution codes are processed on a bit by bit encoding. They are used for implementation in hardware.

User informations are obtained as the input and all the values are converted into ASCII values. They are nothing but 0 's and 1 's . depending upon the modes the values are converted into 0 and 1. There are different types of modes such as numeric , alpha numberic and binary values. The user can give the data in any different modes. Consider the user information as $\mathrm{m} 1, \mathrm{~m} 2, \mathrm{~m} 3$. All these modes are converted in the forms of 0 and 1 .

Now, the input element should be divided into 2 elements of each. The ASCII value of the first element should be added with the ASCII value of the next element and so on. All these values are grouped together to for the block of data as shown in the Figure 3.

After the conversion, these datas such as $\mathrm{m} 1, \mathrm{~m} 2, \mathrm{~m} 3$ are encoded such that the encoded output are divided into 8 bit $\mathrm{s}$ of data. If suppose the data does not contain 8 bit, then zero padding is carried out.

Mask pattern generation is carried out using MATLAB such that the data is highly secured. All the above steps are repeated and entire bit is converted into square blocks. Each and every bit is allocated with the pixel values. Finally the colors such as cyan, yellow and magenta are assigned to all the bits and pixel values. By combining all the colors the final $\mathrm{QR}$ code is obtained in color as shown in the Figure 5

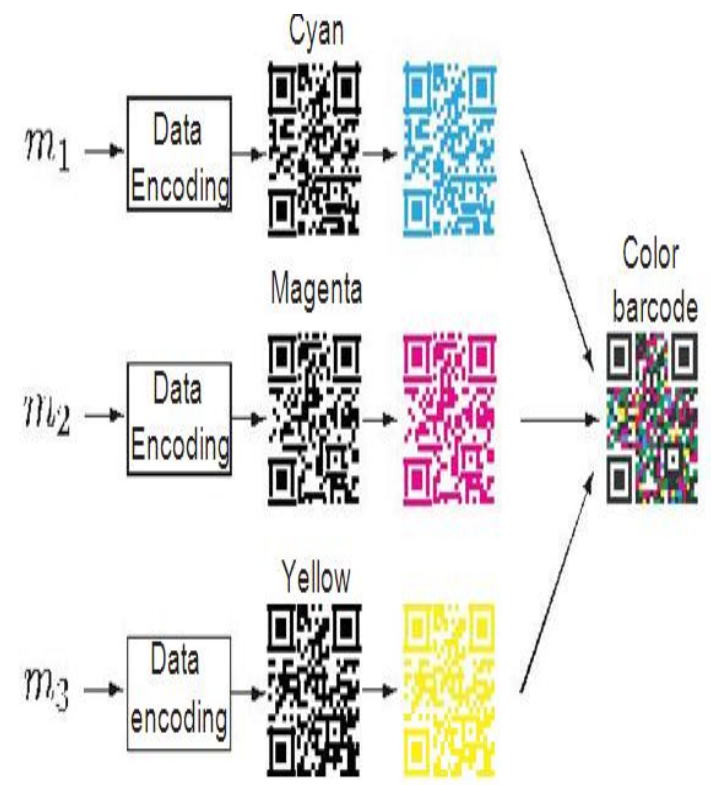

Fig5: Color QR code

The flow chart shown below explains the entire steps for creating the color codes. 


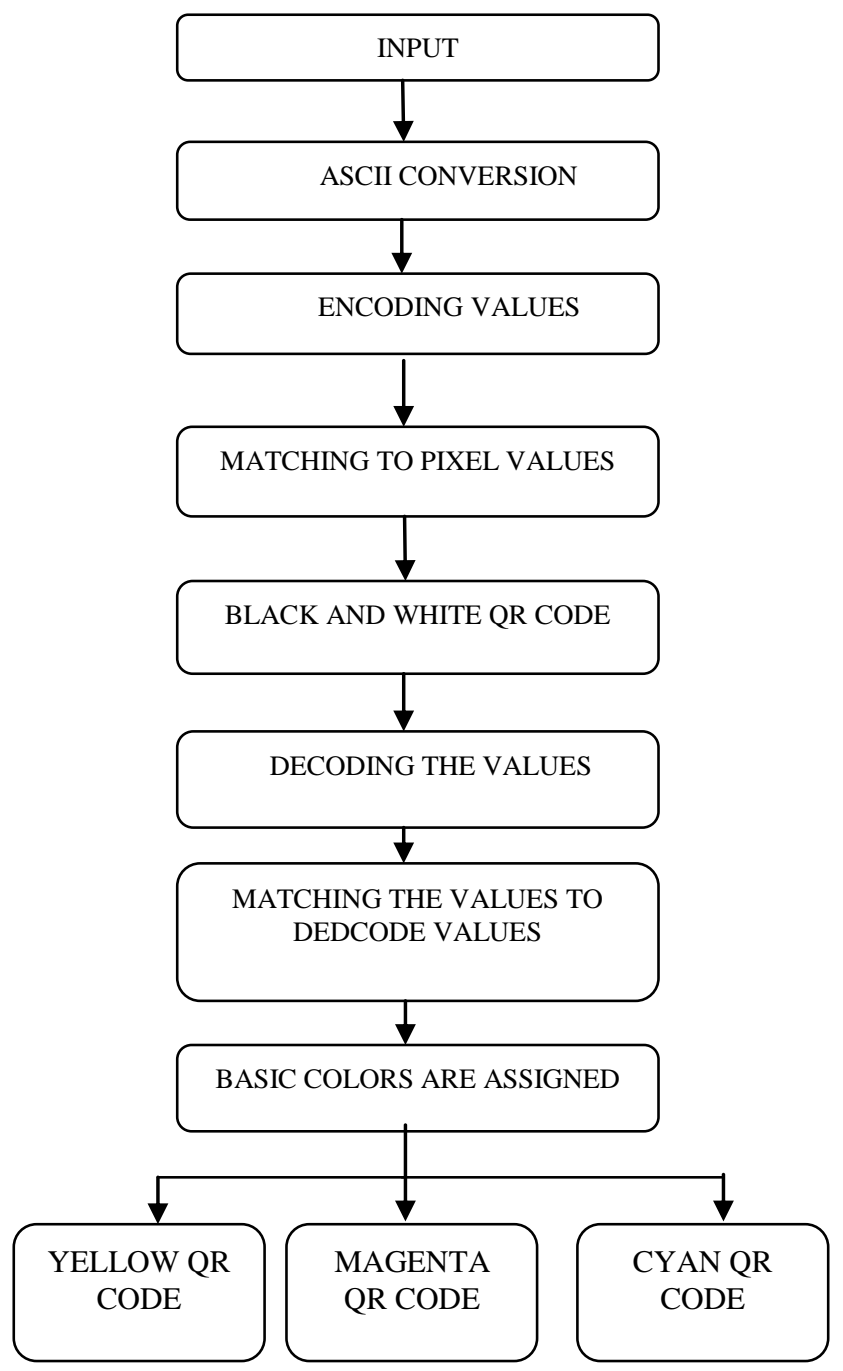

Fig 6: Flow chart for $Q R$ code generation

Here the input is assigned as "Hello World". The input is changed into binary values. These values are encoded with reed Solomon encoder and convolution encoder. The pixel value is assigned for each allocated binary values. Hence Black and white QR code has been obtained from the above encoded values. For the conversion of Black and white QR code into color code, the initial step deal with conversion of encoded code into decoded binary values. Hence the obtained black and white image is decoded and colors are assigned for decoded values. Here cyan color has been assigned for the decoded values.

\section{IMPROVEMENTS IN QR COLOR CODE}

The major advantage for the QR code is shown below:

1. Expand barcode capacity by adding color

- Increase "pixel" size by reducing the number of "pixels" needed to convey information

- Increase the capacity of information in the barcode.

\section{Utilizes existing capabilities of mobile devices}

\section{QR CODE COLOR CHART}

The color chart for $\mathrm{QR}$ code representation has been given in detail along with the bit pattern generation. The combination of
Pixel Values gives different color representation such as black, violet, while, green, blue etc.

\begin{tabular}{|c|c|c|}
\hline$\frac{\text { Color }}{\text { Name }}$ & $\frac{\text { RGB Value }}{\underline{(R, G, B)}}$ & Bit Pattern \\
\hline Black & $0,0,0$ & 000 \\
\hline Red & $255,0,0$ & 001 \\
\hline Green & $0,255,0$ & 010 \\
\hline Yellow & $255,255,0$ & 011 \\
\hline Blue & $0,0,255$ & 100 \\
\hline Violet & $255,0,255$ & 101 \\
\hline Turquoise & $0,255,255$ & 110 \\
\hline White & $255,255,255$ & 111 \\
\hline
\end{tabular}

\section{EXPERIMENTAL RESULTS}

The QR code has been implemented using MATLAB and simulation results are obtained from the MODELSIM. The word "HELLO WORD" is given as the input. The Black and white QR code for the input "hello world" is shown in the Figure 7

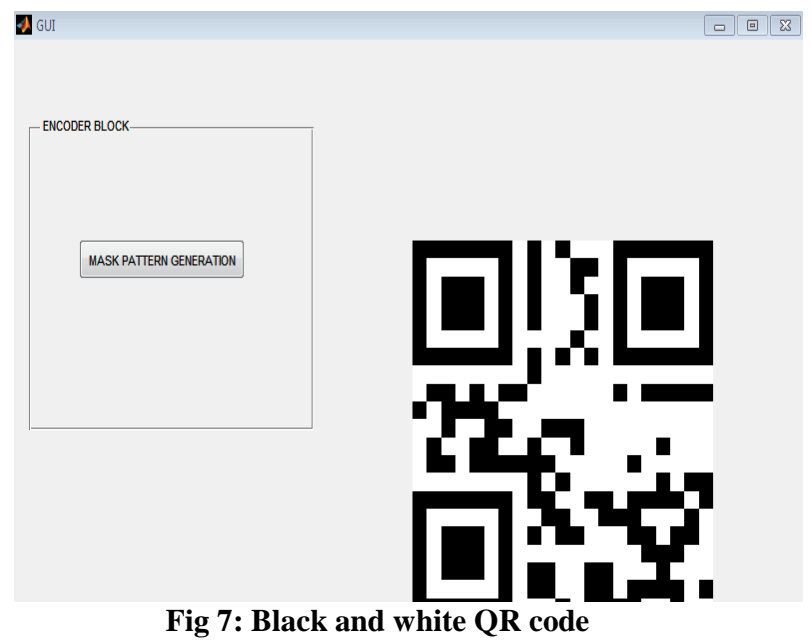

The binary values are obtained fron the MODELSIM which shows the decoded values. Fig 8 represents the decoded image of the black and white QR code. 


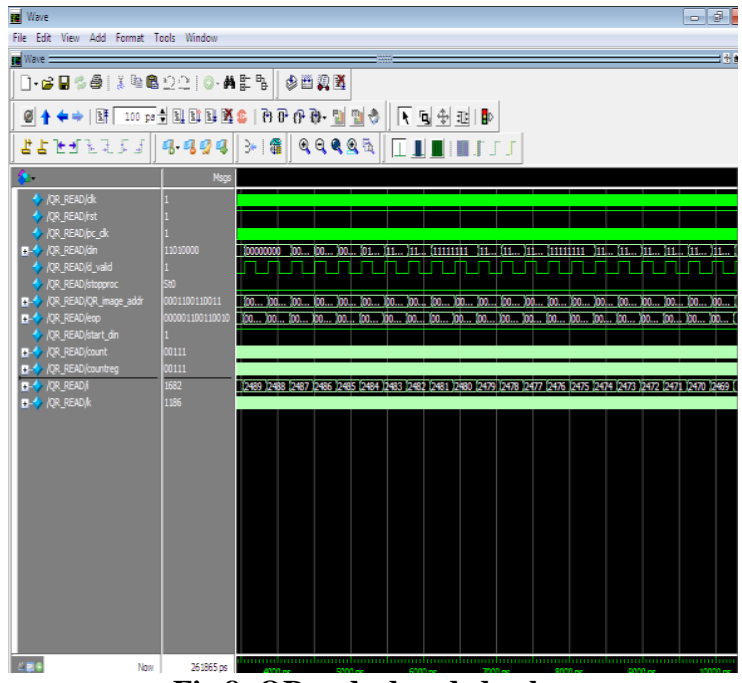

Fig 8: QR code decoded value input.

Fig 9 represents the Cyan color QR code for the provided

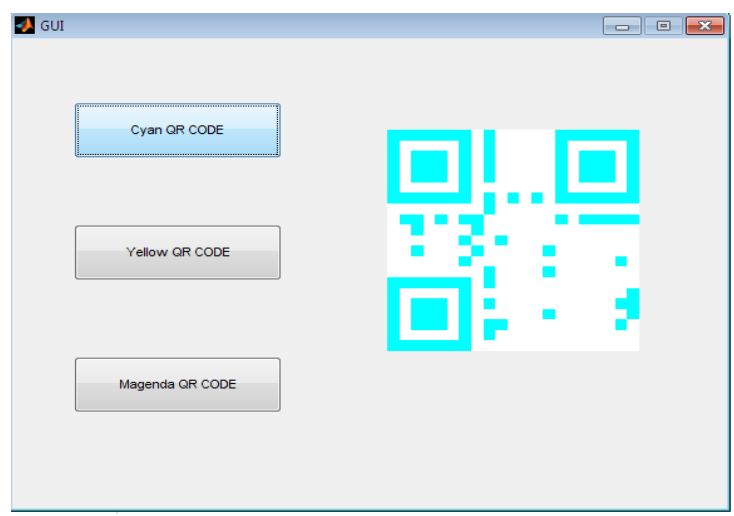

Fig 9: $Q R$ code for Cyan

Fig 10 represents the yellow color QR code for the provided input.

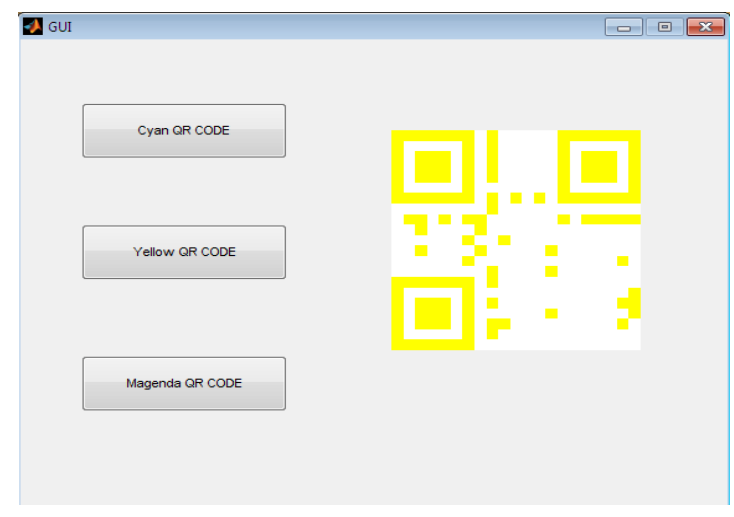

Fig 10: QR code for yellow

Fig 11 represents the Magenta color QR code for the provided input.

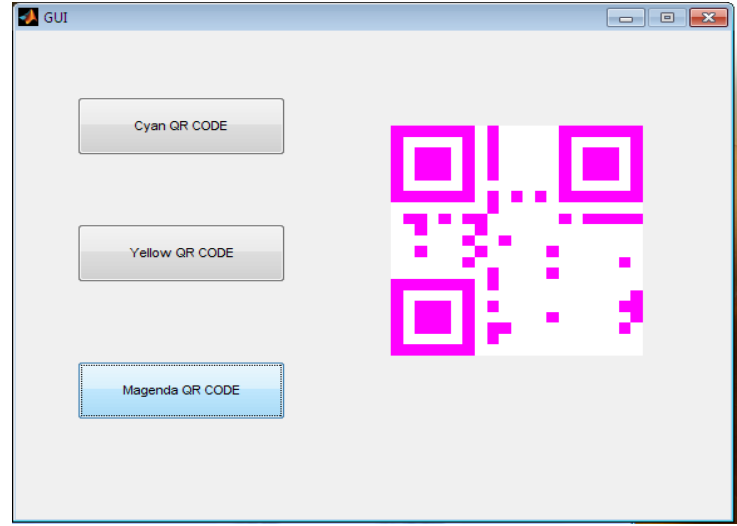

Fig 11: QR code for Magenta

\section{CONCLUSION}

The frame work proposed in this paper provides special techniques for converting the 2D barcode into color codes. It also reads the color code in 360 degree. The obtained QR image has been decoded and binary values of the image are obtained using MODELSIM. The concatenation of all the bits from convolution coder and reed Solomon coder increases the interference cancellation rate and also the embedding capacity. A new high-speed, high-accuracy automatic FPGA based method for generating color QR Code for various text sizes had been proposed. The future work deals with creating other color code such as red, green and blue and also it deals with implementing the color QR code in Real Time applications by using FPGA.

\section{REFERENCES}

[1] Bulan O, H. Blasinski, and G. Sharma Color Qr Codes (2011),' Increased Capacity Via Per-Channel Data Encodingand Interference Cancellation 'Proc. IS\&T/SID 19th Color Imag. Conf., Color Sci. Eng. Syst., Technol.,Appl., Nov., pp. 156-159"

[2] Bulan O, H. Blasinski, and G. Sharma (2010), 'Increased capacity via per-channel data encoding and interference cancellation' IEEE Transaction on image processing Vol. 8.

[3] Bulan O and G. Sharma (2012),'Improved Color Barcodes via Expectation Maximization Style Interference Cancellation': Proc. IEEE Intl. Conf.Acoust. Speech Signal Process., pp. 1509-1512

[4] Henryk Blasinski,Student Member, IEEE (2013),IEEE transactions on image processing, Vol. 22, No. 4.

[5] Orhan Bulan, and Gaurav Sharma, Fellow, IEEE. Precolorant-channel color barcodes for mobile applications: an interference cancellation framewor.

[6] Chi, K.W. Wong (2001), 'A two-stage binarization approach for document images' Proceedings of 2001 International Symposium on Intelligent Multimedia, Video and Speech Processing, May 2-4, pp. 275-278.

[7] GB/T 18284-2000. National standard of the People's Republic of China: Quick Response Code (in Chinese), Issued by China State Bureau of Quality and Technical Supervision.

[8] Gisung Kim, Soobin Lee,Joonyoung Park, Sungkwan Jung, Sangsik Kim, Yong-chul Shin IEEE (2009) 'Thumbnail Selection: Delivering Digital Signage 
Contents to Mobile Phone ' IEEE Transaction on Mobile Computing, Vol. 8, No. 6,

[9] Hara M, M. Watabe, T. Nojiri, T.Nagaya, and Y. Uchiyama (1995),' Optically Readable Two-Dimensional Code And Method And Apparatus Using The Same' U.S. Patent 5726435.

[10] Kim I.J (2004), 'Multi-Window binarization of camera image for document recognition' Proceedings of the 9th Int'l Workshop on Frontiers in Handwriting Recognition,Oct. 26-29, pp.323-327.

[11] ISO/IEC 18004:2000 (2000) Information technologyAutomatic identification and data capture techniques-Bar code Symbology-QR Code.
[12] Mayer, J. ; LPDS Labs. Florianopolis, Brazil ; Bermudez, J.C.M. ; Legg, A.P. ; Uchoa-Filho, B.F.(2009) 'Design Of High Capacity 3d Print Codes Aiming For Obustness To The Ps Channel And External Distortions'Image Processing (ICIP), 16th IEEE International Conference.

[13] Trier O.D,(1995) 'Goal-directed evaluation of binarization methods,'IEEE pami, Vol. 17. No. 12, pp.1191- 1201

[14] Parikh, D. Carnegie Mellon Univ., Pittsburgh, PA, Jancke, G.(2008) 'Localization And Segmentation Of A 2d High Capacity Color Barcode' Proc. IEEE Workshop Appl. Compute pp. 1-6.

[15] Sahoo P.K, S. Soltani, and A.K.C. Wong, (1988) 'A survey of thresholding techniques,' Computer Vision, Graphics, and Image Processing, Vol. 41, pp.233-260. 